\title{
Research and Optimization of Multichannel Wide Dynamic Compression Algorithm in Hearing Aids
}

\author{
Hu Chen ${ }^{1, a)}$, Mingjiang Wang ${ }^{1, b)}$, Mengyan $\mathrm{Li}^{2, c}$, Sixue Xiang ${ }^{2, d)}$ \\ ${ }^{1}$ Harbin Institute of Technology, Shenzhen 518000, China. \\ ${ }^{2}$ Engineering Research Center for Position, Navigation and Time, College of Electronic Science, NUDT, Changsha \\ 410000, China. \\ a) Tiger_tigerchen@163.com \\ b) mjwang@hit.edu.cn \\ c)Ali_limengyan@163.com \\ d) $1205949634 @ q q . c o m$
}

\begin{abstract}
This paper proposes a non-equal width multi-channel loudness compensation algorithm for digital hearing aids. This method achieves a non-equal width multi-channel loudness compensation scheme based on the sensitivity of the human ear to the frequency and the perception of sound intensity. First, the voice signal is moved to the target frequency band in different proportions, and then is divided into non-equal widths, and loudness compensation and gain control are performed in each frequency band. The experimental results show that this method can effectively improve hearing and speech recognition of hearing patients and has a high practical value.
\end{abstract}

Keywords: Non-equal width, Multi-channel, Frequency band, Gain control.

\section{INTRODUCTION}

The key technologies of current digital hearing aids mainly include a loudness compensation algorithm, a noise processing algorithm, and an echo suppression algorithm, all of which aim at improving the wearing experience of a hearing aid [1]. Among these, the loudness compensation algorithm is the core of the key technology. Compared with normal people, the auditory area of patients with hearing impairment is reduced. Therefore, the goal of the wide dynamic compression algorithm is to compress and amplify the sound in the normal human listening domain (eg, 20dB SPL - 100dB SPL), so that the whisper gains more gain, the louder gains less gain, and finally the entire listening domain voice the information maps to the hearing domain of hearing-impaired patients (eg, 50dB SPL $85 \mathrm{~dB}$ SPL) [2]. With further research, it has been found that there is a certain gap between the loudness levels and sound intensity perceived by the human ear in different frequency ranges. Therefore, based on the different listening regions in different frequency ranges, some scholars proposed a multi-channel loudness compensation algorithm. The core idea of this algorithm is to reuse WDRC for each frequency channel based on the wide dynamic compression algorithm. By making the gain factor of each channel different, it is ensured that hearing impaired patients can have a better wearing experience.

\section{MULTI-CHANNEL WIDE DYNAMIC COMPRESSION ALGORITHM AND OPTIMIZATION}

Hearing compensation algorithms are very basic and very important algorithms in hearing aids, and the wide dynamic compression algorithms in hearing compensation algorithms are very common and very important algorithms [3]. The purpose of the algorithm is to compress and amplify the sound signal, and the hearing range of 
the normal person is dynamically compressed into the hearing range of the hearing patient, so that the hearing patient can hear the originally inaudible sound and compress the originally heard sound to Listening feel more comfortable within the range, while ensuring hearing identification and low distortion as shown in Figure 1.

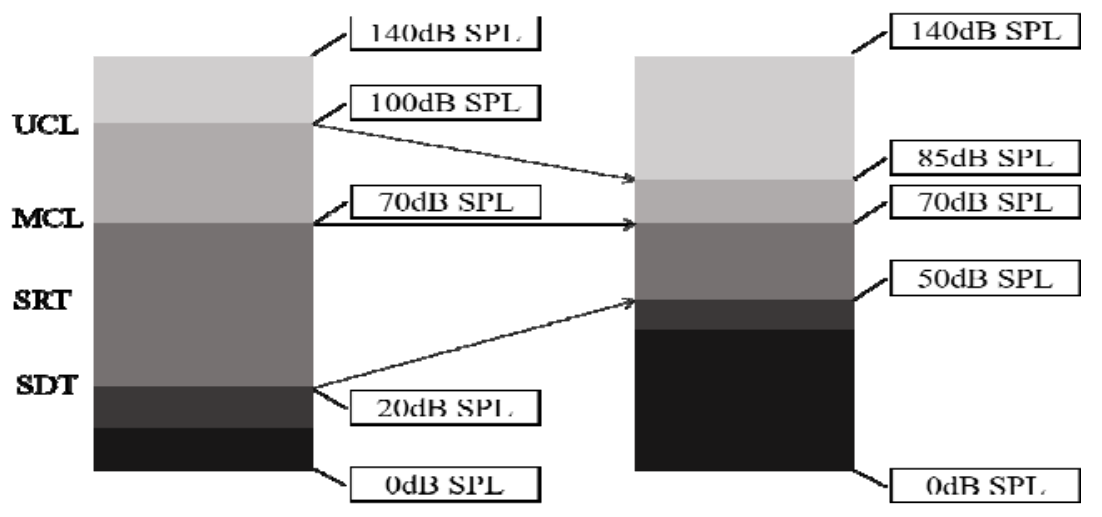

FIGURE 1. The Principle of Wide Dynamic Compression Algorithm

\section{Sliding Mode Observer Design}

Because the sound field in real life does not change all the time, the sound pressure at each moment is called the instantaneous sound pressure. For convenience, we usually calculate the sound pressure value over time, as shown in equation (1).

$$
P_{e}=\sqrt{\frac{1}{T} \int P^{2} d t}
$$

Although the effective sound pressure value is the sound pressure that we usually refer to, but in reality, we cannot measure continuous sound pressure values and only get some discrete points. In this case, we take a segment of sound whose duration is $\mathrm{T}$ and discrete points are N. as shown in equation (2).

$$
\begin{gathered}
P_{e}=\sqrt{\frac{1}{T} \sum_{n=1}^{N} P^{2} \Delta t}=\sqrt{\frac{1}{N \Delta t} \sum_{n=1}^{N} P^{2} \Delta t}=\sqrt{\frac{1}{N} \sum_{n=1}^{N} P^{2}} \\
L_{p}=20 \log _{10}\left(\frac{p}{p_{\text {ref }}}\right)(d B S P L)
\end{gathered}
$$

Sounds are converted to electrical signals through the microphone through the amplifier circuit, analog-to-digital converter (A/D) to allow the processor to process, so the sound pressure of the microphone collected voice signal and the actual sound pressure level exists for certain differences, we assume that they differ by a $\mathrm{D}$ factor.

Then, the spectrum X (n) can be obtained by performing a discrete Fourier transform on the input signal X (n). Then, calculating a channel sound pressure level with a length of $\mathrm{K}$ can be calculated by:

$$
P^{2}=\frac{1}{N \times M} \sum_{i}^{K}\left(|X(i)|^{2}+|\tilde{X}(i)|^{2}\right)
$$




$$
L=20 \log _{10}\left(\frac{\sqrt{P^{2}}}{P_{r e f}}\right)
$$

In a hearing aid, the actual input signal after the signal passes through the microphone and the analog to digital converter is:

$$
\begin{aligned}
& x^{\prime}(n)=D \times x(n), X^{\prime}(n)=D \times X(n) \\
& L^{\prime}=20 \log _{10}\left(\frac{\sqrt{(D \times P)^{2}}}{P_{\text {ref }}}\right)=20 \log _{10}\left(D \times \frac{\sqrt{P^{2}}}{P_{r e f}}\right) \\
& =20 \log _{10}\left(\sqrt{P^{2}}\right)-20 \log _{10}\left(P_{r e f}\right)+20 \log (D) \\
& =L+E
\end{aligned}
$$

In this article, we use the decibel meter and the digital hearing aid to record six different voice signals in the same environment [4]. Then we calculate the sound pressure level difference between the decibel meter and the hearing aid in the six groups of audios, and then the six groups of sound pressure values. The average value of the difference between the decibel meter and the hearing aid was $7.12 \mathrm{~dB}$.

\section{Parametric Multi-Channel Division}

The basement membrane in the human ear plays a very large role in the choice of frequency. The lower the frequency of the sound, the smaller the loss during transmission and the longer the transmission distance. Based on experiments and tests, people finally obtained the relationship between base film position and characteristic frequency:

$$
\begin{aligned}
& f=\int_{0}^{x} \Delta f_{c b}=A\left(10^{a x}-K\right) \\
& f=165.4\left(10^{0.06 x}-0.88\right)
\end{aligned}
$$

Next we need to divide the frequency of the basement membrane into non-equal width channels. Studies have shown that the frequency range of $20 \mathrm{~Hz}$ to $16 \mathrm{kHz}$ can be divided into 24 critical characteristics similar to the critical band based on the human ear's perception of frequency, as in equation (10):

$$
i=\frac{26.81 \hat{f}_{i}}{1960+\hat{f}_{i}}-0.53, i=1,2,3, \ldots
$$

The number of channels divided will affect the effect of compensation. The traditional channel division is generally three, and we know that the more the number of channels divided, the better the compensation effect, but the same amount of calculation and power will be greater [5]. Due to the limitations of digital hearing aid power and computational capabilities, we next divided the number of channels into eight, and the results are shown in Table 1. 
TABLE 1. Channel Division Result Table

\begin{tabular}{|c|c|c|c|}
\hline Channel & Lower border(Hz) & Upper border(Hz) & Bandwidth(Hz) \\
\hline 1 & 0 & 630 & 630 \\
\hline 2 & 630 & 1080 & 450 \\
\hline 3 & 1080 & 1480 & 400 \\
\hline 4 & 1480 & 2000 & 520 \\
\hline 5 & 2000 & 2320 & 320 \\
\hline 6 & 2320 & 3150 & 830 \\
\hline 7 & 3150 & 5260 & 2110 \\
\hline 8 & 5260 & 8000 & 2740 \\
\hline
\end{tabular}

\section{Loudness Compensation}

FIG. 2 corresponds to the optimal threshold of a normal person and the optimal threshold of a patient. It can be seen from the figure that the hearing threshold of the hearing patient is higher than that of the normal person, and the pain threshold is lower than that of the normal person [6]. The compression ratio CRa and the compression ratio $\mathrm{CRb}$ of the segment $\mathrm{b}$ can be obtained by calculation:

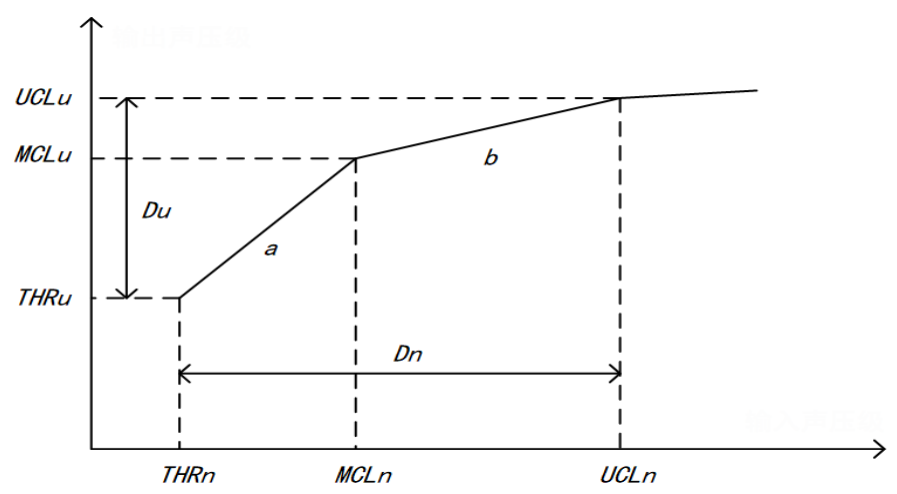

FIGURE 2. Wide Dynamic Compression Algorithm Curve

According to the compression ratio and the input sound pressure level, the output sound pressure level expression can be obtained:

$$
\text { SPLout }=\left\{\begin{array}{c}
\text { SPLin, SPLin }<\text { THRn } \\
\text { THRu }+\frac{\text { SPLin }-T H R n}{C R a}, T H R n \leq S P L i n<M C L n \\
M C L u+\frac{\text { SPLin }-M C L n}{C R b}, M C L n \leq \text { SPLin }<U C L n \\
U C L n, \text { SPLin } \geq U C L n
\end{array}\right.
$$

Assuming that there are $\mathrm{n}$ points $(\mathrm{x} 1, \mathrm{f1}),(\mathrm{x} 2, \mathrm{f} 2), \ldots,(\mathrm{xn}, \mathrm{fn})$ in the plane, we need to construct a polynomial $\mathrm{p}(\mathrm{x})$ to fit a curve through the $n$ points. Set the interpolation node to $x i$ and the function value is fi, $i=0,1 \ldots, n$. Let the interpolation polynomial $\mathrm{P}(\mathrm{x})$ have the following form:

$$
P_{n}(x)=a_{0}+a_{1}\left(x-x_{0}\right)+a_{2}\left(x-x_{0}\right)\left(x-x_{1}\right)+\cdots+a_{n}\left(x-x_{0}\right)\left(x-x_{1}\right) \cdots\left(x-x_{n}-1\right)
$$

The interpolation condition is $i=0,1 \ldots, n$. 
If we divide the range of input $20 \mathrm{~dB} \sim 120 \mathrm{~dB}$ into three segments, the first segment is $20 \mathrm{~dB} \sim 40 \mathrm{~dB}$ with 1 st-order interpolation; the second segment is $40 \mathrm{~dB} \sim 90 \mathrm{~dB}$ with 2 nd-order interpolation; the third segment is $90 \mathrm{~dB} \sim 120 \mathrm{~dB}$ with 1 Interpolation [7].

$$
\begin{aligned}
& \operatorname{gain}_{S P L}=S P L_{\text {out }}-S P L_{\text {in }} \\
& \text { gain }=10^{\text {gain }_{S P L} / 20}
\end{aligned}
$$

From formula (14) we can calculate the gain of each channel.

\section{SIMULATION}

We use an audio verification with a sampling frequency of $16 \mathrm{kHZ}$ and a time of about $3 \mathrm{~s}$. Suppose there is a patient with high frequency loss and low frequency loss. Now we first simulate the original signal of this segment of speech, and then pass this audio through the multi-channel wide dynamic compression algorithm of this article and then print out the loudness-compensated audio amplitude. The effectiveness of the algorithm is verified by comparing the gain of the signal after the loudness compensation. FIG. 3 is the original waveform of the normalized speech signal, and FIG. 4 is the waveform diagram of the speech signal after the loudness compensation.

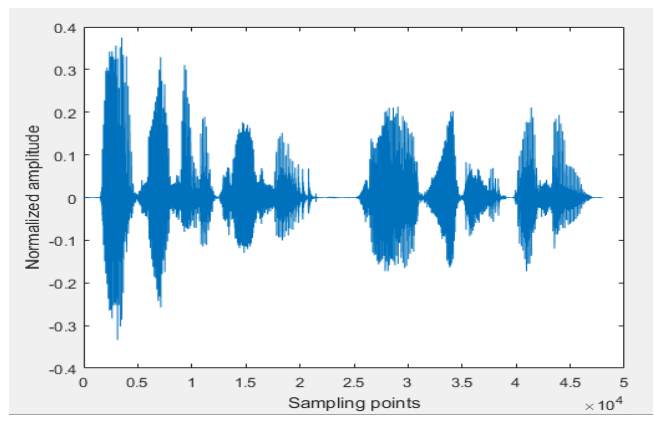

FIGURE 3. Multi-Channel Wide Dynamic Compression Waveform before Audio

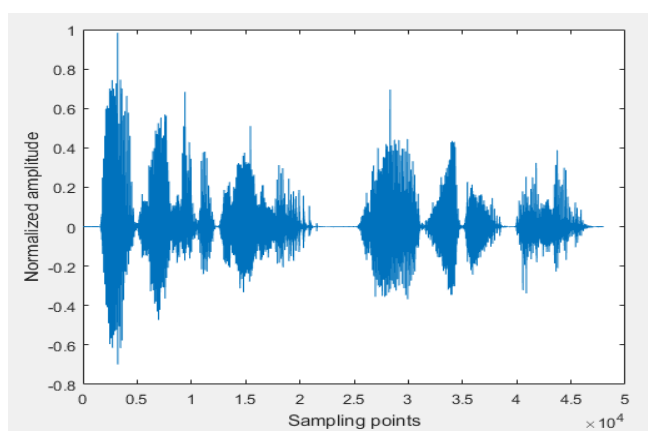

FIGURE 4. Multi-Channel Wide Dynamic Compressed Audio Waveform

From Figure 3 and Figure 4, we can find that after the multi-channel wide dynamic compression loudness compensation algorithm is compensated, the overall envelope of the voice signal remains better, and the amplitude of the audio at each sampling point is also compensated to varying degrees effect. 


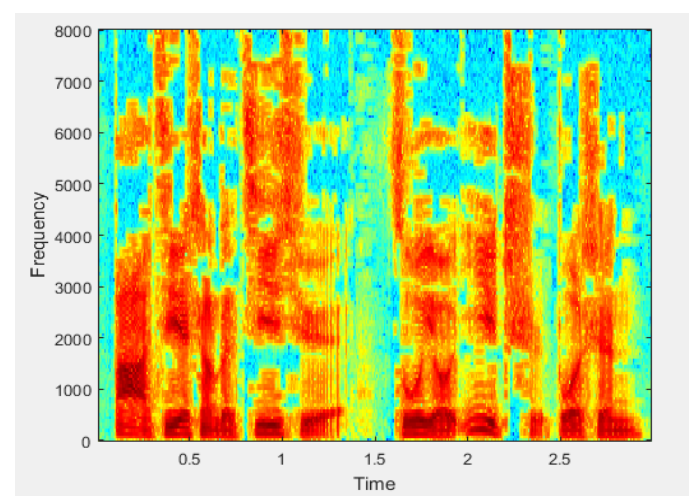

FIGURE 5. Before the Speech Spectrogram Compensation

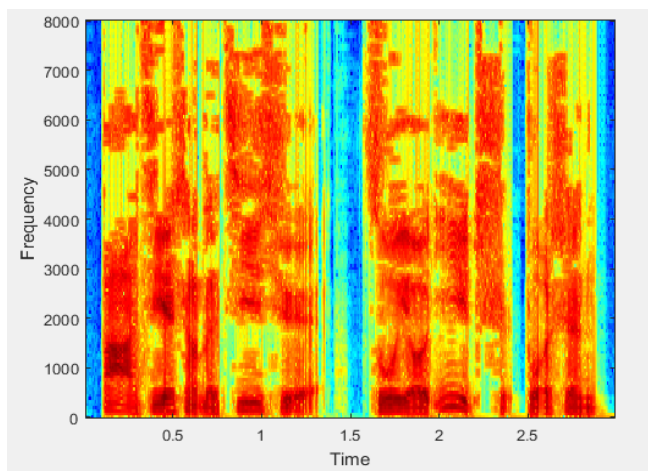

FIGURE 6. After the Speech Spectrogram Compensation

Fig. 5 and Fig. 6 are the speech spectra before and after speech compensation. The coordinate value of the spectral map is the energy of the speech data. The size of the energy is expressed by the color, and the deeper the energy is, the stronger the energy is. Comparing the two graphs, the compensated speech energy is higher than the pre-compensation speech. Although the energy is still mainly concentrated in the low-frequency region, after compensation, the energy at high frequencies is obviously increased, while at low frequencies there is not much difference, which is in line with the high-frequency loss of the patient's demand gain.

Next, we select a certain frame of speech signal. Here we select the speech signal of the 100th frame. The gain curve of the loudness compensation of this signal is shown in Fig. 7. It can be seen that after a loud dynamic compression loudness compensation algorithm. The gain of the speech signal at each frequency point changes, and the gain in the high frequency part is much greater than the low frequency, which meets the compensation requirements for severe patients with high frequency loss.

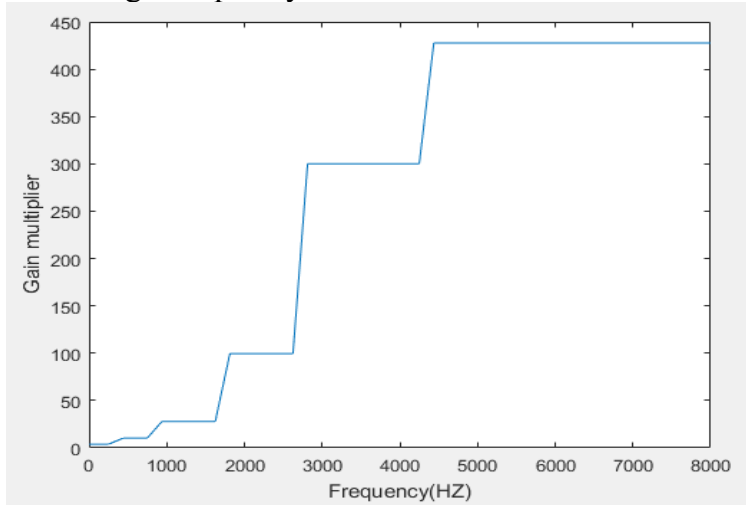

FIGURE 7. Compensation Gain Curve 


\section{CONCLUSION}

This paper provides a reference for the multi-channel division in frequency domain by analyzing the human ear's perception of frequency. The rules of channel division are analyzed. According to the processing methods in hearing aids, the channels are divided into parameters, the number and length of channels are defined by parameters, and the flexibility of use in hearing aids is enhanced. Then according to the auditory condition of the patient's various frequencies, an appropriate compensation curve is generated. The compensation curve is generated by a Newton interpolation method through a known discrete point. Finally, an audiogram of a hearing-impaired patient was used as an example to simulate the algorithm. A standard Chinese Mandarin test voice is input, and the speech spectral graphs before and after loudness compensation are compared. The algorithm can effectively compensate different frequency signals according to the hearing condition of the patient.

\section{REFERENCES}

1. Hamacher V, Chalupper J, Eggers J, et al. Signal Processing in High-End Hearing Aids: State of the Art, Challenges, and Future Trends [J]. Eurasip Journal on Applied Signal Processing, 2005, 2005(18):2915-2929.

2. Schneider T, Brennan R. A multichannel compression strategy for a digital Hearing aid[C]. IEEE International Conference on Acoustics, Speech, and Signal Processing. IEEE, 2002:411.

3. Levitt H. Noise reduction in hearing aids: a review [J]. Journal of Rehabilitation Research \& Development, 2001, 38(1):111.

4. Chung K. Challenges and recent developments in hearing aids. Part I. Speech understanding in noise, microphone technologies and noise reduction algorithms [J]. Trends in Amplification, 2004, 8(3):83.

5. Schum, Donald J. Noise reduction via signal processing: (1) Strategies used in other industries [J]. Hearing Journal, 2003, 56(5):27-32.

6. Serizel R, Moonen M, Wouters J, et al. A speech distortion weighting-based approach to integrated active noise control and noise reduction in hearing aids [J]. Signal Processing, 2013, 93(9): 2440-2452.

7. Mojiri M, Karimi-Ghartemani M, Bakhshai A. Processing of Harmonics and Interharmonics Using an Adaptive Notch Filter [J]. IEEE Transactions on Power Delivery, 2010, 25(2):534-542. 